Saudi Journal of Medical and Pharmaceutical Sciences

Abbreviated Key Title: Saudi J Med Pharm Sci ISSN 2413-4929 (Print) |ISSN 2413-4910 (Online) Scholars Middle East Publishers, Dubai, United Arab Emirates Journal homepage: https://saudijournals.com/sjmps

\title{
Exudative Age-Related Macular Degeneration: A Case Report and Review of the Literature
}

Imane Chabbar ${ }^{*}$, Louai Serghini, Abdelkrim Boulanouar, Amina Berraho

Ophthalmology B Department, Ibn-Sina University Hospital, Rabat, Morocco

DOI: $10.36348 /$ sjmps.2020.v06i06.010 $\quad$ | Received: 16.06 .2020 | Accepted: 24.06 .2020 | Published: 30.06 .2020

*Corresponding author: Imane Chabbar

\section{Abstract}

Age-related macular degeneration (AMD) is a progressive degenerative retinal disease with particular damage in the macular area. It is the most common cause of blindness in developed countries mainly affecting the elderly. The exudative or wet form represents the most severe form with rapid progression despite therapeutic progress. It is characterized by the development of choroidal neovessels, crossing the retinal pigment epithelium and developing below the macular area. We report a clinical case of exudative AMD complicated by macular edema and serous retinal detachment, diagnosed in a 70-year-old patient with cardiovascular risk factors and presenting reduced visual acuity with central scotoma. Through this case we study the risk factors, the diagnostic and therapeutic procedure of exudative AMD with a review of the literature.

Keywords: Age-related macular degeneration AMD; risk factors; diagnosis; anti-VEGF; treatment.

Copyright @ 2020: This is an open-access article distributed under the terms of the Creative Commons Attribution license which permits unrestricted use, distribution, and reproduction in any medium for non-commercial use (NonCommercial, or CC-BY-NC) provided the original author and source are credited.

\section{INTRODUCTION:}

Age-related macular degeneration is a degenerative macular disease occurring in genetically predisposed patients [1]. This pathology develops as a function of various innate and environmental factors. Its frequency is increasing due to the life expectancy increase. It has become the main cause of blindness in developed countries in elderly patients over 50 years old [2]. Thanks to the development of retinal and choroid imaging techniques, the management of agerelated macular degeneration is improving. Age-related macular degeneration is divided into two types: dry or atrophic form and wet or exudative form. Currently, only exudative AMD receives treatment to slow the disease progression. However, this pathology remains incurable, whatever its type. We report a clinical case of exudative AMD through which we discuss risk factors, diagnostic and therapeutic procedure with a review of the literature.

\section{Case Report}

A 74-year-old male patient, hypertensive for 10 years with smoking cessation for 8 years, presented for a rapidly progressive visual acuity decrease in the right eye associated with a central scotoma, one month ago. The ophthalmological examination showed best corrected visual acuity at counting fingers in the right eye and $8 / 10$ in the left eye. A decrease in near vision with reading inability was also noted. The examination of the anterior segment was normal apart from a cortical cataract in both eyes. The right eye fundus examination (Figure 1) reveals a clear vitreous, chorioretinal atrophy and a reshaped and raised macular area with absent foveal reflex and perimacular hemorrhages.

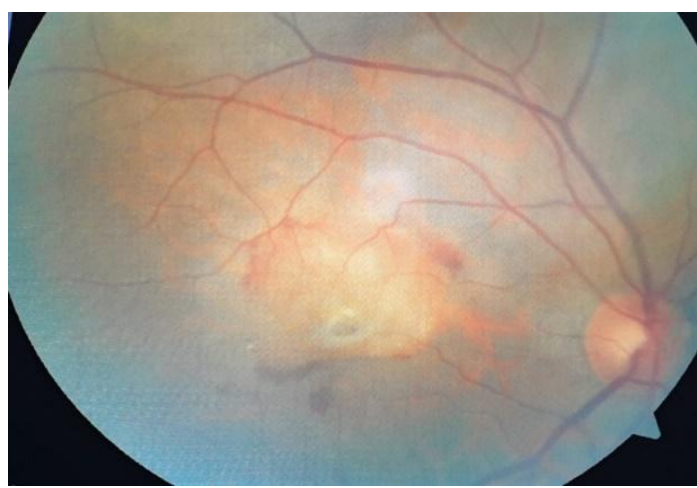

Fig-1: Color fundus retinal photography of the right eye showing diffuse chorioretinal atrophy, a reshaped and raised macular area with absent foveal reflex and perimacular hemorrhages

Retinal Fluorescein Angiography (Figure 2) showed early hyperfluorescence in the macular area, gradually becoming intense with diffusion in favor of visible neovessels, and perimacular hypofluorescence due to mask effect of hemorrhages. 


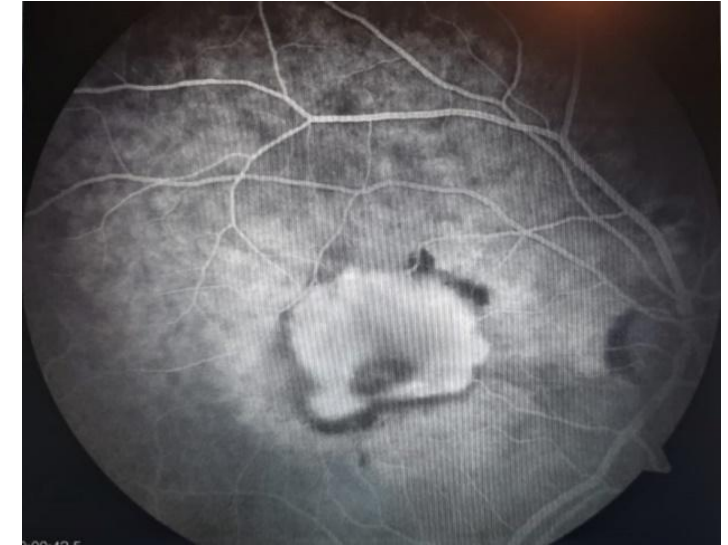

Fig-2: Fluorescein Angiography of the right eye showing an early hyperfluorescence in the macular area, gradually becoming intense with diffusion in favor of visible neovessels, and perimacular hypofluorescence due to mask effect of hemorrhages
Macular optical coherence tomography (OCT) (Figure 3) objectified an additional sub-retinal hyperreflectivity with an alteration of the different retinal layers: disorganization and detachment of retinal pigment epithelium, cystoid macular edema and serous retinal detachment suggesting choroidal neovessels.

The diagnosis of advanced exudative AMD was retained and treatment with intravitreal injections of anti-VEGF was indicated. The seriousness of the macular lesions pleads in favor of a poor prognosis. Thus, strict surveillance of the adelphe eye seems essential.
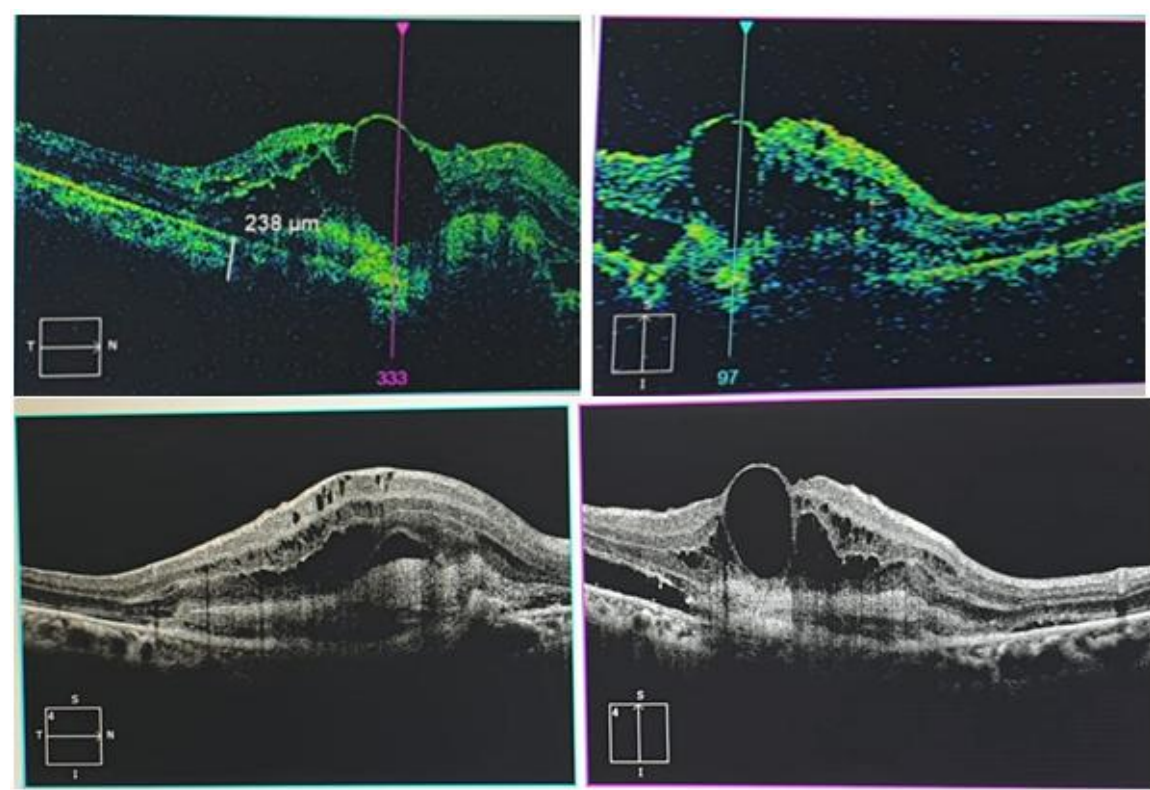

Fig-3: Macular OCT showing an additional subretinal hyperreflectivity with an alteration of the different retinal layers: disorganization and detachment of retinal pigment epithelium, cystoid macular edema and serous retinal detachment suggesting choroidal neovessels.

\section{Discussion AND REVIEW OF THE}

\section{LITERATURE}

Age-related macular degeneration is a degenerative macular disease occurring in genetically predisposed patients. It is the first cause of low vision in developed countries for elderly people over 50 years old. It is a disabling condition characterized by a progressive loss of central vision [1,2]. Exudative AMD represents approximately $10 \%$ of cases. It is the most severe form with rapid progression despite therapeutic progress. It is characterized by the development of choroidal neovessels, crossing the retinal pigment epithelium and developing below the macular area [3]. There are two main types of subretinal neovessels: Visible neovessels with rapid development, causing severe vision loss. However, these are the least common and only affect $15 \%$ of people with neovessels. Occult neovessels are more frequent but slowly evolving. Macular OCT is a very useful tool for detecting the presence of exudative manifestations: direct signs such as visible neovessels with localized thickening of the deep layer, or indirect signs such as intraretinal edema, serous retinal detachment or retinal pigment epithelium detachment [4]. Fluorescein Angiography allows to differentiate both forms of choroidal neovessels. Indocyanine Green Angiography can also be requested in addition. In the absence of treatment, macular retinal detachment, exudates and subretinal hemorrhages cause a destruction of photoreceptors and as a result a severe decrease in visual acuity with permanent central scotoma. The evolution is then marked by a fibrous scar with progressive atrophy of chorioretinal tissues. The real causes of AMD are not yet fully established, but certain risk factors have been identified by researchers. Some are not controllable and others can be changed [5]. As its name suggests, this macular degeneration is 
primarily related to age, which represents the biggest risk factor for this disease. The majority of patients are over the age of 50 and with each older age group the proportion of patients increases. Ocular factors could be associated, such as hypermetropia, pallor of the iris or even cataract surgery. Cardiovascular factors have also been identified [6]. Indeed, high blood pressure and dyslipidemia represent risk factors for exudative AMD. Patients with exudative AMD are 4 times more likely to have high blood pressure than patients without AMD. Several studies have shown that with age, fatty acids and cholesterol accumulate at the Bruch's membrane. These deposits can interfere with retinal pigment epithelium functions and therefore promote the development of AMD [6,7]. Regarding genetic factors, some studies have been able to demonstrate the hereditary nature of AMD and the responsibility for certain genes mutations in its appearance $[8,9,10]$. The female sex is more affected, more particularly in case of early menopause, suggesting the hypothesis of estrogenic protection against the development of AMD. Other studies have shown that blindness secondary to AMD is rare in black people. The protective factor of choroidal melanin against oxidative stress allows to explain these results [2]. Regarding environmental factors involved, active smoking multiplies by 34 the risk of developing AMD. Passive smoking also exposes to an additional risk. Tobacco reduces circulating antioxidants and thus retinal protection during UV exposure. It also alters choroidal blood flow and nicotine promotes neovascularization [11]. Other studies have also shown the impact of excessive exposure to blue waves and the implication of deficiency in oligoelements and vitamins [12].

Therapeutically, AMD is a chronic disease requiring regular monitoring and appropriate therapy. First, disease prevention by reducing controllable risk behaviours such as UV protection, adapted diet and quitting smoking. Taking antioxidant vitamin supplements may slow progression [13]. Once the diagnosis of exudative AMD is made, a well-coded treatment must be quickly implemented. The techniques of thermal laser retinal photocoagulation and dynamic phototherapy have been mainly replaced by antiangiogenic injections implemented in 2006. Thermal laser photocoagulation was one of the first treatments for exudative AMD. The laser interacts with the retinal pigment epithelium and helps prevent the neovessels extension to other healthy retinal areas, but it leaves a permanent non-functional retinal scar. Thus, the indications for this "destructive" photocoagulation are limited to the treatment of extrafoveal or juxtafoveal neovessels in order to prevent their extension towards the macular center without direct effect on the gain of visual acuity [13]. Dynamic phototherapy is currently limited to the treatment of exudative AMD with visible retrofoveal neovascularization, but is no longer the first-line treatment. In addition, it can be used in case of contraindication or non-response to anti-VEGF and in certain clinical forms in combination with anti-VEGF [13]. Currently, intravitreal injections of anti-VEGF are the most effective and widely used method for the treatment of exudative AMD, reducing the proliferation of choroidal neovessels [14]. These drugs are more effective in the active phases of neovessels development, however, their interest is very limited in scarred or overly advanced forms. The indications of first-line anti-VEGF intravitreal injections in the treatment of exudative retrofoveal AMD are: a decrease in visual acuity (greater than or equal to 5 letters with the presence of edema in OCT), an increase in central macular thickness more than $100 \mu \mathrm{m}$ (macular edema in OCT), a persistent edema in OCT (causing serous retinal detachment) [15]. After the injection, a monthly monitoring is sufficient if total drying is observed. However, a new injection is necessary in the case of exudative recurrence. If the drying is durable, a spacing of controls frequency is envisaged according to the "treat and extend" method in order to reduce the number of injections and controls.

\section{CONCLUSION}

Age-related macular degeneration is the means cause of blindness in developed countries, representing a real public health problem. It depends on many factors and appears after 50 years. Its frequency is increasing more and more with the longer life expectancy and new daily habits (tobacco, diet). Through this case report, we reviewed the risk factors and the diagnostic and therapeutic management of exudative AMD. Indeed, several recent studies have allowed a significant advance in the knowledge of this disease thus improving its management. Atrophic AMD is currently untreated. However, the treatment of exudative AMD has known an important advance with the appearance thermal laser retinal photocoagulation techniques, followed by dynamic phototherapy, replaced since 2006 by intravitreal anti-angiogenic injections which represent an essential therapy to slow the neovessels proliferation.

\section{Conflict of interest}

The authors declare that they have no conflict of interest.

\section{REFERENCES}

1. Ratnapriya, R., \& Chew, E. Y. (2013). Agerelated macular degeneration-clinical review and genetics update. Clinical genetics, 84(2), 160-166.

2. Smith, W., Assink, J., Klein, R., Mitchell, P., Klaver, C. C., Klein, B. E., ... \& de Jong, P. T. (2001). Risk factors for age-related macular degeneration: pooled findings from three continents. Ophthalmology, 108(4), 697-704.

3. Ferris, F. L., Davis, M. D., Clemons, T. E., Lee, L. Y., Chew, E. Y., Lindblad, A. S., ... \& Klein, R. 
(2005). A simplified severity scale for age-related macular degeneration: AREDS Report No. 18. Archives of ophthalmology (Chicago, Ill.: 1960), 123(11), 1570-1574.

4. Mylonas, G., Ahlers, C., Malamos, P., Golbaz, I., Deak, G., Schütze, C., ... \& Schmidt-Erfurth, U. (2009). Comparison of retinal thickness measurements and segmentation performance of four different spectral and time domain OCT devices in neovascular age-related macular degeneration. British journal of ophthalmology, 93(11), 1453-1460.

5. Chakravarthy, U., Wong, T. Y., Fletcher, A., Piault, E., Evans, C., Zlateva, G., ... \& Mitchell, P. (2010). Clinical risk factors for age-related macular degeneration: a systematic review and metaanalysis. BMC ophthalmology, 10(1), 31.

6. Klein, R., Klein, B. E., Knudtson, M. D., Wong, T. Y., Cotch, M. F., Liu, K., ... \& Jacobs Jr, D. R. (2006). Prevalence of age-related macular degeneration in $4 \mathrm{racial} / \mathrm{ethnic}$ groups in the multiethnic study of atherosclerosis. Ophthalmology, 113(3), 373-380.

7. Munch, I. C., Linneberg, A., \& Larsen, M. (2013). Precursors of age-related macular degeneration: associations with physical activity, obesity, and serum lipids in the inter99 eye study. Investigative ophthalmology \& visual science, 54(6), 3932-3940.

8. Spencer, K. L., Olson, L. M., Schnetz-Boutaud, N., Gallins, P., Agarwal, A., Iannaccone, A., ... \& Scott, W. K. (2011). Using genetic variation and environmental risk factor data to identify individuals at high risk for age-related macular degeneration. PloS one, 6(3), e17784.

9. Gorin, M. B. (2012). Genetic insights into agerelated macular degeneration: controversies addressing risk, causality, and therapeutics. Molecular aspects of medicine, 33(4), 467-486.

10. Priya, R.R., Chew, E.Y., Swaroop, A.(2012). Genetic studies of age-related macular degeneration: lessons, challenges, and opportunities for disease management. Ophthalmology, 119: 2526-2536.

11. Kabasawa, S., Mori, K., Horie-Inoue, K., Gehlbach, P. L., Inoue, S., Awata, T., ... \& Yoneya, S. (2011). Associations of cigarette smoking but not serum fatty acids with age-related macular degeneration in a Japanese population. Ophthalmology, 118(6), 1082-1088.

12. Chew, E. Y., Clemons, T. E., Agrón, E., Sperduto, R. D., SanGiovanni, J. P., Kurinij, N., ... \& AgeRelated Eye Disease Study Research Group. (2013). Long-term effects of vitamins C and E, $\beta$ carotene, and zinc on age-related macular degeneration: AREDS report no. 35. Ophthalmology, 120(8), 1604-1611.

13. Hernández-Zimbrón, L. F., Zamora-Alvarado, R., Velez-Montoya, R., Zenteno, E., Gulias-Cañizo, R., Quiroz-Mercado, H., \& Gonzalez-Salinas, R. (2018). Age-related macular degeneration: new paradigms for treatment and management of AMD. Oxidative medicine and cellular longevity, 2018.

14. Brown, D. M., Kaiser, P. K., Michels, M., Soubrane, G., Heier, J. S., Kim, R. Y., ... \& Schneider, S. (2006). Ranibizumab versus verteporfin for neovascular age-related macular degeneration. New England Journal of Medicine, 355(14), 1432-1444.

15. CATT Research Group. (2011). Ranibizumab and bevacizumab for neovascular age-related macular degeneration. New England journal of medicine, 364(20), 1897-1908. 